\title{
Optimized Route Finding in GPS Navigation Using KALMAN'S Filter Algorithm
}

\author{
Gayathri.S ${ }^{1}$, Ajith.P2 ${ }^{2}$ Balasubramaniyam .CR ${ }^{3}$, Bharath.E ${ }^{4}$ \\ ${ }^{1}$ Assistant Professor, Department of Computer Science, Rajalakshmi Institute of Technology, Chennai \\ ${ }^{2,3,4}$ UG, Department of Computer Science, Rajalakshmi Institute of Technology, Chennai
}

\begin{abstract}
This project applies two AI (Artificial Intelligence) techniques, i.e., fuzzy logic and expert system, to enhance the Kalman's filterbased GPS Navigation System. The Dijktra's Algorithm to find the smart route to reach the destination. The Kalman's Filtering technology provides us the exact speed of the vehicle by means of an accelerometer and a speed sensor. Once the speed has been calculated, the algorithm uses the speed of the vehicle and converts the speed to RPM (Revolution per minute) of the wheel to find the traffic intensity. Once the traffic has been noted in a particular area it sends the information to the centralised database. The database in turn notifies the user and Police Department about the traffic and once the traffic has been identified in the path of the user, the algorithm finds an alternate path by considering factors like traffic and time to reach the destination. The notifications are sent to the user and Police Department by means of SMTP and POP3 protocol. Since the Police Department are notified with the traffic intensity, the Expert Systems checks if the traffic has been cleared or not and sends all the updates to the centralised databases.
\end{abstract}

Keywords: Fuzzy Logic, Kalman's Filtering Technology, Expert Systems, Dijkstra's Algorithm, POP3 and SMTP protocols.

\section{INTRODUCTION}

A small step towards ARTIFICIAL INTELLIGENCE can make a great change in our life. One is to travel smart by knowing the shortest route, travelling at a low cost, with an immensively faster rate. If we are about to make a travel, we initially uses our application to get the smart route to reach the destination. Initially the Algorithm searches for the shortest path to reach our destination. Once the path is provided by the algorithm the user starts to make his travel on the same path. During travel, the algorithm executes at a particular interval say, 10minutes, the algorithm checks that the route that we are travelling is free from traffic or traffic loaded and whether the path remains to be the shortest path in the position of the user. The position of the user is identified by GPS through an active internet connection. The traffic at a particular place can be found by calculating the rotational count of the wheel by knowing the speed, calculated from kalman's filter ${ }^{[1]}$. The traffic intensity is found by making a conditional check of the rotational count for a particular cycle. If traffic was reported at a

particular place then the algorithm checks for database for an alternate route and sends that information via notifications to the user and Police Department. The Expert System waits till the police takes necessary steps to clear the traffic and makes the updates to the database... Once the alternate route has been identified, the algorithm checks if the new path is free from traffic and the time required to reach the destination is as equal to the former one. After all these process, the algorithm sends all these information to the centralised databases.

\section{RELATED WORKS}

Artificial Intelligence techniques like Fuzzy Logic ${ }^{[2]}$ and Expert Systems ${ }^{[3]}$ have been used for GPS Navigation. Kalman's Filter is the major tool that is used to produce output in noisy systems. Kalman's Filter finds its applications in Tracking Objects, Fitting Bezier patches to point data, Economics, Navigation, Computer vision applications like feature tracking, cluster tracking, fusing data from radar and laser sensor and velocity measurements. Fuzzy logic Systems is mainly used in the area of noisy receptions where the input data was only of crisp values. This method finds its applications in Automatic gearboxes, four wheel steering, Vehicle Environment Control, Hi-fi Systems and Photocopiers. Dijkstra's Algorithm ${ }^{[4]}$ is mainly used to find the shortest path between two areas.

This algorithm is already used in vast areas like Google Maps.

The integration of Fuzzy logic along with kalman's filter ${ }^{[1]}$ for calculating the exact speed of the vehicle is the main property involved in finding out the traffic intensity of the vehicle. Since a conventional speedometer only provides a nominal speed of the moving vehicle, it becomes practically impossible for us to calculate the traffic intensity. By 


\section{International Advanced Research Journal in Science, Engineering and Technology}

Vol. 8, Issue 5, May 2021

DOI: $10.17148 /$ IARJSET.2021.8584

knowing the exact speed from this Algorithm we could possibly calculate the rotational count of the wheel. Once the count of the wheel has been calculated the algorithm execute recursively through the traffic intensity can be found out.

\section{EXISTING SYSTEM}

In the current scenario, GPS navigations that are used by the Google maps intend only to find the shortest path by using dijkstra's algorithm ${ }^{[4]}$. Several other algorithm are also used in finding the shortest path but not efficient as the former algorithm. According to statistical data, Navigation Systems in car form a great part in global navigation market, with 180 million car navigation systems being installed worldwide in 2013. AMONG these 110 million were PNDs and about 70 million were factory installed or added after market. However PND's are increasingly replaced by smart phones which comes with built in navigation systems or some other applications that needs to be purchased. Google and Apple maps are being used by 68.6 million peoples worldwide. In this scenario it becomes immensively simple for this algorithm to be integrated into the GPS systems of all the land vehicles.

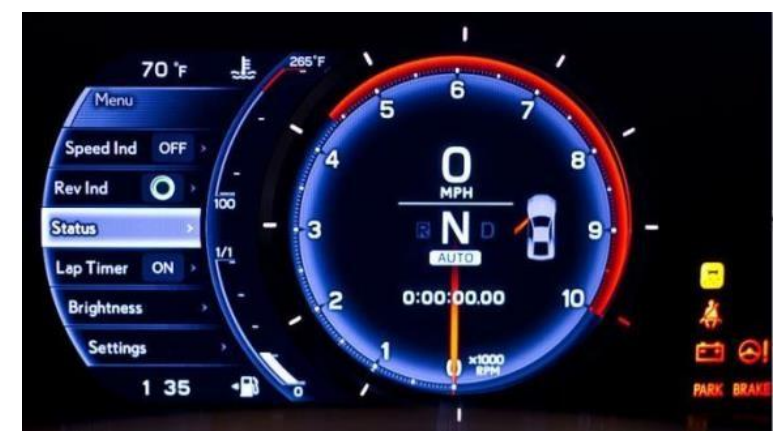

However a speedometer ${ }^{[5]}$ provides the speed of the vehicle only by means of estimation.

The values that are given as input are just a rounded off numbers which are hardly difficult for our process to be used. The process that is involved in calculating the speed of the vehicle by means of a speedometer is diagrammatically explained below,

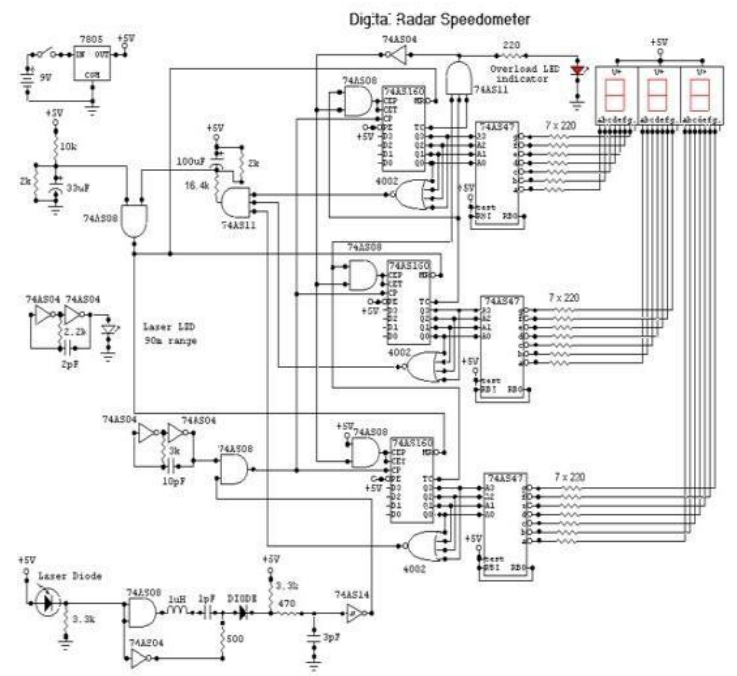

Kalman's Filtering Technology is being used to get an exact output for the data taken under consideration. With this technology many technological application finds the solution for their system by means of considering various experimental values and providing the crisp input values. As far as Navigation is concerned this technology is currently being used in finding the location and speed of the vehicle by means of integrating this technology with Fuzzy logic System. Fuzzy Systems is the major reason for providing the exact solution while integrating it with Kalman's Filtering Technology. Many concepts like Multi sensor systems, Robot location and vehicle navigation for identifying speed use this kind of technology in two sections. Firstly Fuzzy systems provides an overall set of values that are needed for the calculation of the solution whereas Kalman's filter separates up all the erratic disturbances and provides and exact path for calculating the exact solution whatever the scenario may be. 


\section{International Advanced Research Journal in Science, Engineering and Technology}

Vol. 8, Issue 5, May 2021

DOI: $10.17148 /$ IARJSET.2021.8584

\section{PROPOSED DESIGN:}

Initially the user chooses a specific place as destination. Once the user chooses the destination, the GPS checks for their location. Once the Algorithm gets the location of the user and destination, it checks the database for the smart route free from traffic and less time that take to reach the destination by making use of Dijkstra's Algorithm. All these informations can be accessed from the centralised databases. While the user is on the way to reach their destination, the Algorithm executes at a particular interval and checks if there o0ccurs any traffic along the path. Simultaneously the speed of the vehicle will be calculated and the revolution speed of the wheel can be calculated by means of the formula RPM = Circumference of the wheel * Speed of the vehicle (in minutes) Circumference will be calculated by knowing the radius of the wheel. The speed of the vehicle can be calculated exactly by means Fuzzy logic based Kalman's Filtering Technology. As the revolution count of the wheel will be calculated at particular intervals, if the revolution count decreases beyond an average then the area can be marked as congested area. And if the path of the user is being congested, the notifications are sent to the user and police department via POP3 and SMTP protocol. The Expert Systems keep track of the location of the user and the information conveyed to the Police Department.

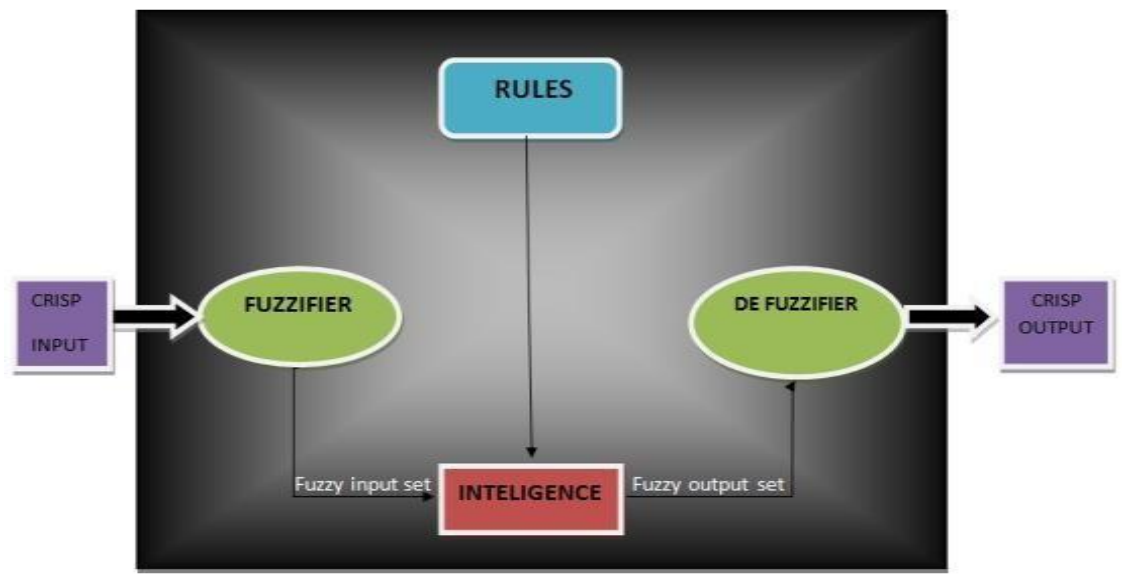

Dijkstra's Algorithm checks for other alternate paths to reach the destination by considering the factors like congestion, quicker route and less time. The notifications sent to the Police Department are kept in track with the expert Systems and will be updated to the database if the traffic was cleared or not.

\section{FLOWCHART}

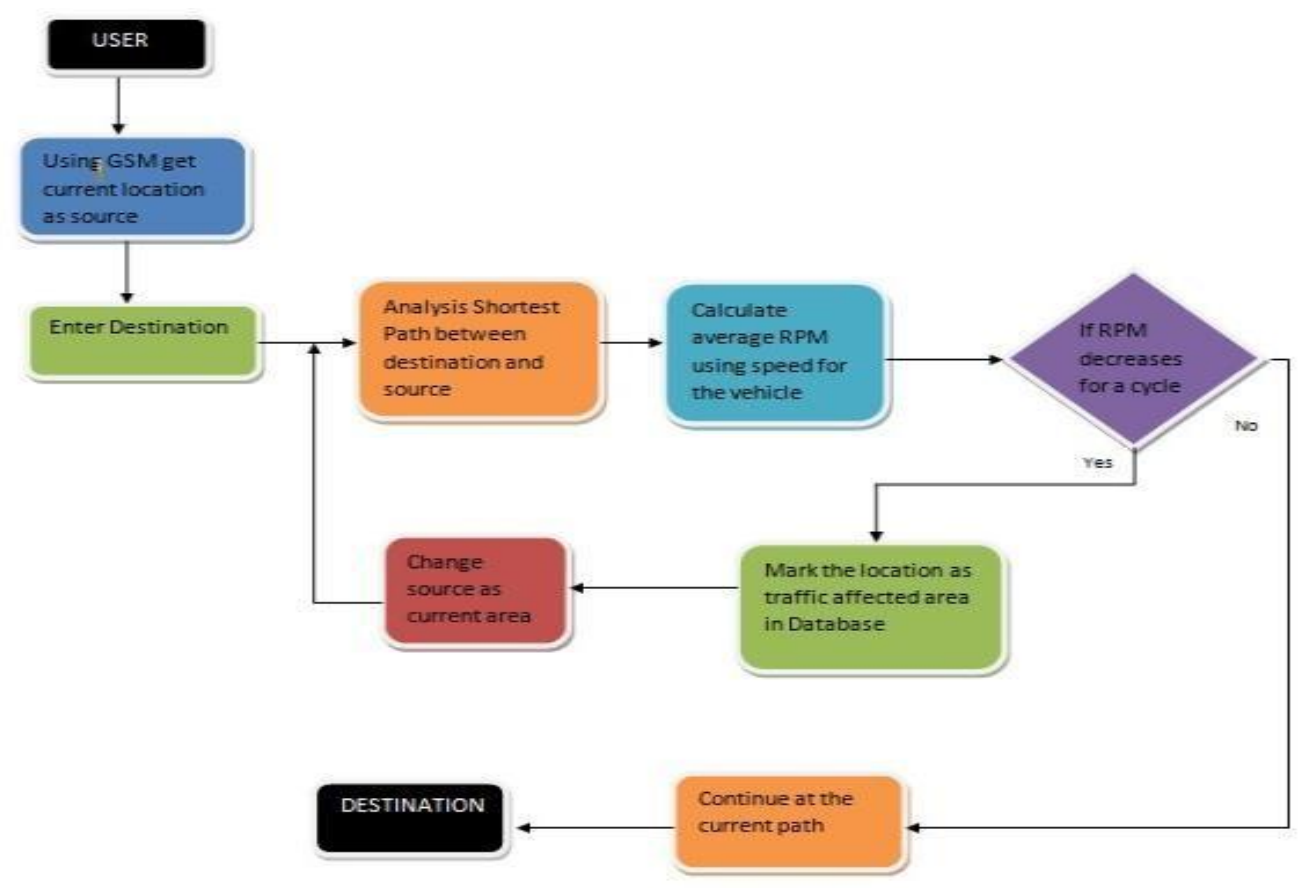


International Advanced Research Journal in Science, Engineering and Technology

Vol. 8, Issue 5, May 2021

DOI: $10.17148 /$ IARJSET.2021.8584

\section{EXPERIMENTAL ANALYSIS}

An experimental analysis with the present existing model of GPS navigation and the proposed model with Artificial Intelligence based GPS navigation is shown below.

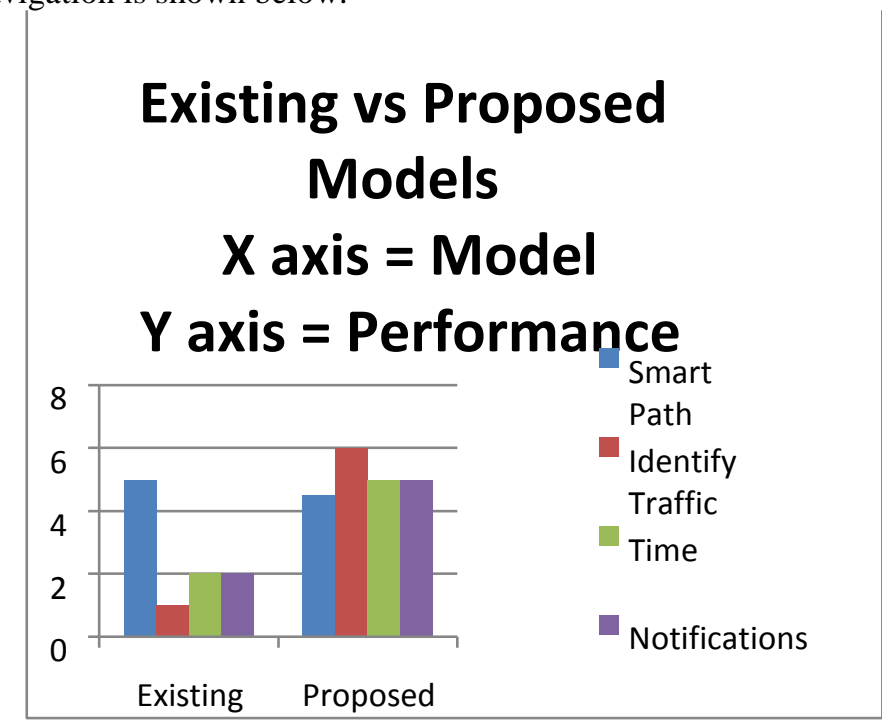

\section{ARCHITECTURE DIAGRAM}

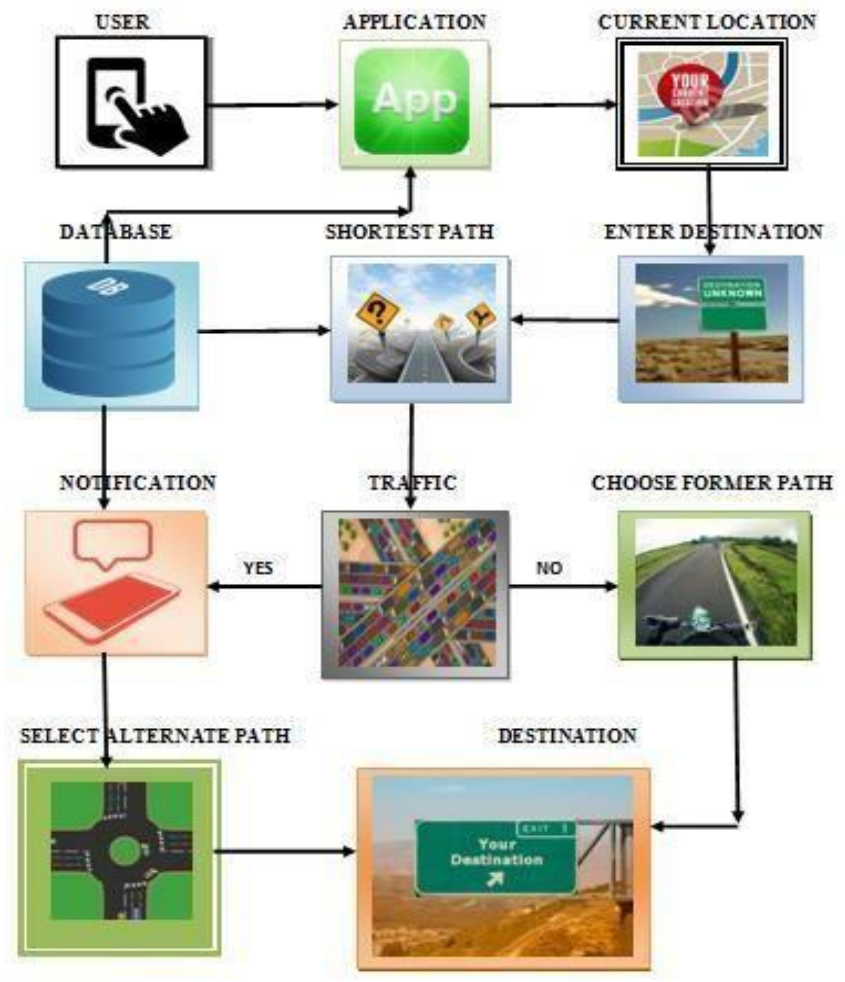

VII. CONCLUSION

The existing ideas about GPS navigation only circulates in the area of finding the quicker way using shortest path algorithms without considering the time and traffic intensity. In this developing world these two are the major factors that should be taken into account for vehicle navigation. By implementing this project, we can overcome factors like time and traffic to find the smart path to reach our destination. 


\section{International Advanced Research Journal in Science, Engineering and Technology}

Vol. 8, Issue 5, May 2021

DOI: $10.17148 /$ IARJSET.2021.8584

\section{REFERENCES}

[1] Estimation of absolute vehicle speed using fuzzy logic rule-based Kalman's filter Conference Paper · July 1995

[2] Web Reference: http://www.tutorialspoint.com/artificial_inte lligence/fuzzy_logic/

[3] Expert Systems for fault Diagnosis in Engineering Applications, IEE Colloquium on 20 April 1989.

[4] Web Reference: https://en.wikipedia.org/wiki/Shortest__path algorithms

[5] Web Reference: http://stackoverflow.com/questions/1557054 2/determining-the-speed-of-a-vehicleusinggps-in-android

[6] WebReference:https://en.wikipedi a.org/wiki/Fuzzy_control_system

[7] WebReference:https://www.statista.com/topics/2221/navigation-devicesandusage/

[8] Development of a fuzzy logicbased adaptive Kalman's filter (http://ieeexplore.ieee.org/)

[9] WebReference:https://en.wikipedia.org/wiki/Kalman_filter

[10] Intelligent System Design and Engineering Application (ISDEA), 2010 International Conference on 13-14 Oct. 2010

[11] Control and Decision Conference (CCDC), 2013 25th Chinese 10.1109/CCDC.2013.6560927.

[12] Intelligent Systems and Applications, 2009.ISA 2009. International Workshop on 23-24 May 2009 10.1109/IWISA.2009.5072990

[13] Sensors, 2010 IEEE10.1109/ICSENS.2010.5690738.

[14] Information Fusion (FUSION), 2012 15th International Conference on9-12 July 2012.

[15] Sustainable Utilization and Development in Engineering and Technology (STUDENT), 2011 IEEE Conference on 20-21 Oct. 2011 10.1109/STUDENT.2011.6089337

[16] Design of Reliable Communication Networks, 2003. (DRCN 2003) Proceedings Fourth International Workshop on 19-22 Oct. 2003 10.1109/DRCN.2003.1275389

[17] Computational Intelligence and Software Engineering, 2009. CiSE 2009. 10.1109/CISE.2009.5364482

[18] Broadband Network \& Multimedia Technology, 2009. IC-BNMT '09. 2nd IEEE 10.1109/ICBNMT.2009.5348490.

[19] IEEE Transactions on Biomedical Engineering (Volume: 63, Issue: 10, Oct. 2016) 10.1109/TBME.2015.2510365

[20] IEEE Transactions on Intelligent Transportation Systems (Volume: PP, Issue: 99) 10.1109/TITS.2016.2606369 